\title{
Neonatal pasteurellosis: a review of reported cases
}

\author{
N Nakwan, ${ }^{1}$ N Nakwan, ${ }^{2}$ T Atta, ${ }^{3}$ K Chokephaibulkit ${ }^{4}$
}

${ }^{1}$ Neonatal Intensive Care Unit, Department of Pediatrics, Hat Yai Hospital, Songkhla,

Thailand; ${ }^{2}$ Department of Medicine, Hat Yai Hospital, Songkhla, Thailand;

${ }^{3}$ Department of Pediatrics,

Trang Hospital, Trang, Thailand;

${ }^{4}$ Division of Infectious Diseases, Department of Pediatrics,

Mahidol University, Bangkok,

Thailand

\section{Correspondence to:}

Narongsak Nakwan, Neonatal Intensive Care Unit, Department of Pediatrics, Hat Yai Medical

Education Center, Hat Yai Hospital, Songkhla 90110,

Thailand; nnakwan@hotmail. com

Accepted 2 February 2009

Published Online First

10 February 2009

\begin{abstract}
Background: Pasteurellosis is an uncommon infectious disease in humans mainly caused by Pasteurella multocida infection in neonates and has been rarely reported.

Objectives: To review the literature and address the mode of transmission, clinical presentation, laboratory diagnosis, treatment, outcome and potential risk factors related to neonatal pasteurellosis.
\end{abstract}

Methods: A Medline all-languages database search for neonatal (birth-1 month) pasteurellosis cases after 1950 was conducted. Individual references from each publication were also reviewed to identify additional cases.

Results: Thirty-two cases were found, but detailed information was available for this review in only 25 cases. The median age was 14 days (range: birth-30 days). All were infected with $P$ multocida. Animal exposure to cats and/or dogs was the major risk of infection: non-traumatic exposure in 11 (44\%) cases, and traumatic exposure in 2 $(8 \%)$ cases. Infections in 11 (44\%) cases were classified as vertical transmission. The clinical features were most commonly bacteraemia with or without meningitis. The age at onset of $72 \mathrm{~h}$ or older was significantly associated with meningitis ( $\geqslant 72 \mathrm{~h}$ of age: $13 / 14$ vs $<72 \mathrm{~h}$ of age: $3 / 11$, $p=0.002$ ). The most used antibiotics were $\beta$-lactam with or without aminoglycoside or chloramphenicol. The overall mortality was $20 \%(5 / 25)$. The age at presentation of $<72 \mathrm{~h}$, birth weight of $<2500 \mathrm{~g}$, and vertical transmission were independently associated with death.

Conclusion: Pasteurellosis is a rare bacterial infection in neonates and should be considered in the cases of sepsis with history of exposure to domestic animal in either the patient or the mother.

Pasteurellosis is an uncommon infectious disease in humans and caused mainly by Pasteurella multocida. ${ }^{12}$ Pasteurella spp. is Gram-negative coccobacilli oral flora found in many kinds of animals, especially cats $(70-90 \%)$, dogs $(50-66 \%)$ or pigs $(51 \%) .{ }^{1} P$ multocida infection in neonates is a serious systemic disease, leading to meningitis, septicaemia, pneumonia, conjunctivitis, and osteoarticular infection. ${ }^{3-27}$ The infections are mostly acquired through scratches and bite wounds from domestic animals. ${ }^{128}$ Vertical transmission is another route of infection reported in neonates. ${ }^{4} 58$ 11-13 15-17 2427

This article reviewed the literature of neonatal pasteurellosis from 1953 to the present, to address the mode of transmission, clinical presentation, laboratory diagnosis, treatment, outcome and potential risk factors related to mortality.

\section{METHODS}

We searched the PubMed bibliographic database (US National Library of Medicine, Bethesda, Maryland, USA) in the period of 1950 to March 2008 using the key words "newborn: birth-1 month", "pasteurellosis", "pasteurella infection" and "Pasteurella spp.",

\section{What is already known on this topic}

- Neonatal pasteurellosis is a rare but serious bacterial infection with relatively high mortality.

\section{What this study adds}

- Sepsis and meningitis are common in neonatal pasteurellosis. Most of the infections in neonates occurred without traumatic exposure to domestic animals.

- Early-onset sepsis, birth weight, and vertical route of infection are significant factors related to mortality in neonatal pasteurellosis.

with no language restrictions. After this initial series of reports was reviewed, the individual references listed in each publication were again reviewed to find additional case reports.

For this analysis, diagnosis of a definite case of pasteurellosis required isolation of Pasteurella spp. in organ(s) and/or body fluid in a neonate. Vertical transmission was defined as documented evidence of Pasteurella spp. infection in organ(s) and/or body fluid in the mothers, and/or the patients developed pasteurellosis within $72 \mathrm{~h}$ of age and without history of neonatal exposure to animals. Traumatic exposure was defined as being bitten or scratched by the animals, and non-traumatic exposure was defined as direct contact and/or indirect contact with animals without evidence of bite or scratch. The demographic parameters, clinical presentations, treatments and outcomes of the patients were collected from each case report, and extracted onto a data extraction form by two independent investigators ( $N$. Narongwit and A. Thitiya). Relevant data from French and German language articles were extracted in the presence of a translator. The SPSS program (V.11.5, SPS Inc, Chicago, Illinois, USA) was used to develop a database of categorical and continuous variables. Statistical evaluation of differences in proportions was performed by Fisher's test. A p value of less than 0.05 indicated statistical significance.

The Hat Yai Research Ethics Committee was contacted and declared that no ethical approval was required for this research.

\section{RESULTS}

A total of 32 cases were identified in the original search, but detailed clinical data were available of the analysis in only 25 cases. The earliest case was reported in 1953, and the last case reported in February 2008. Table 1 summarises the main features of the 25 reported neonatal cases. All were infected by $P$ multocida. 
Table 1 Demographic and clinical characteristics of 25 reported cases of neonatal pasteurellosis sorted by year of publication

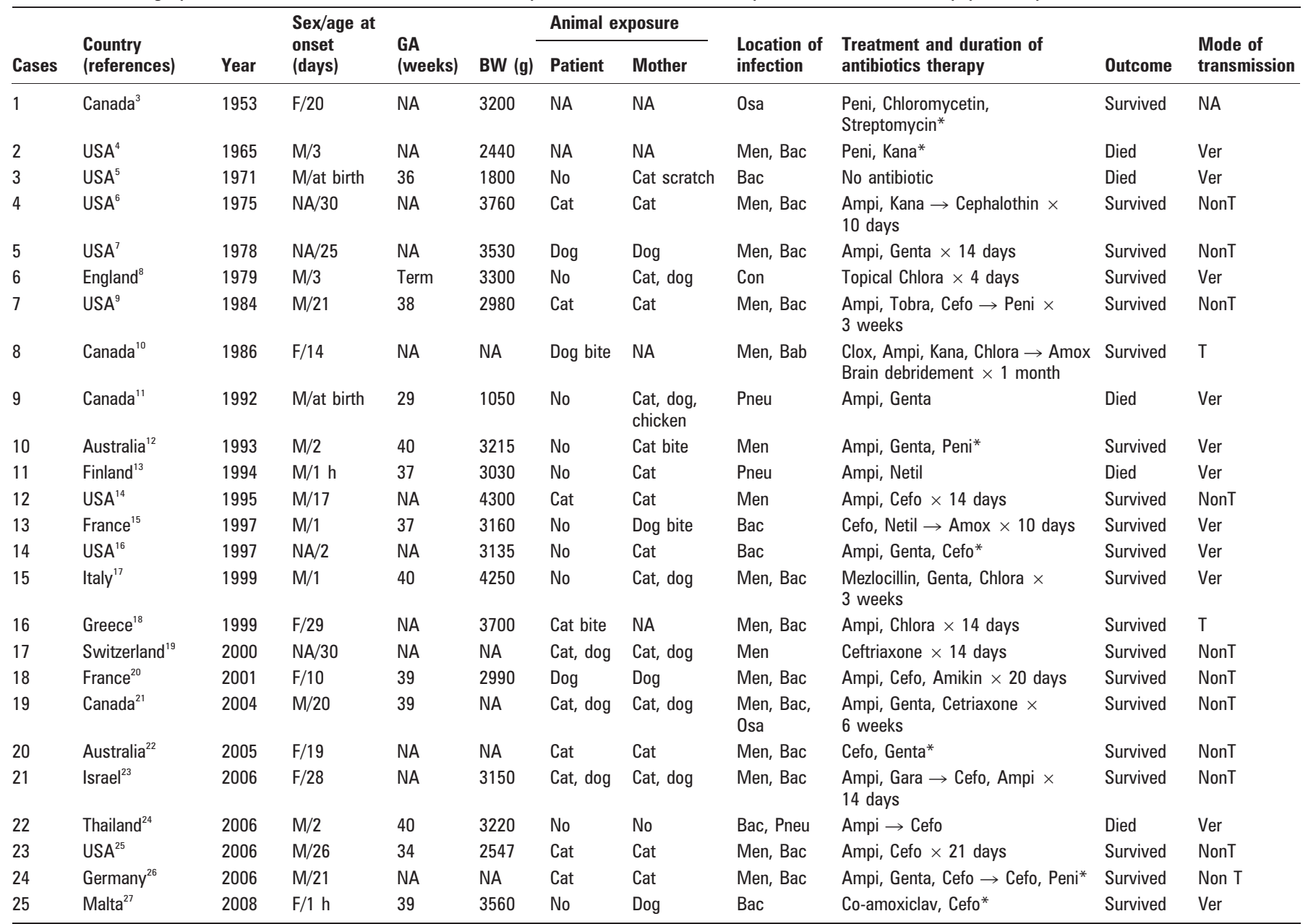

$\rightarrow$ Indicates changing antibiotics. ${ }^{*}$ The duration of antibiotic treatment was not available.

Amox, amoxicillin; Ampi, ampicillin; Bab, brain abscess; Bac, bacteraemia; BW, birth weight; Cefo, cefotaxime; Chlora, chloramphenicol; Clox, cloxacillin; Con, conjunctivitis; F, female; GA, gestation age; Gara, garamicin; Genta, gentamicin; Kana, kanamycin; M, male; Men, meningitis; NA, not available; Netil, netilmicin; NonT, non-traumatic; Osa, osteomyelitis; Peni, penicillin; Pneu, pneumonia; T, traumatic; Tobra, tobramycin; Ver, vertical transmission.

\section{MODE OF TRANSMISSION}

Of the 25 reported cases, 23 reported the information of animal exposure. Non-traumatic animal exposure was a major risk of infection, and was reported in $11(44 \%)$ cases (six, two and three cases exposed to cats, dogs and both, respectively). Traumatic animal exposure was reported in two cases, one of those (case 8) had developed meningitis and brain abscess following a dog bite at the scalp, while another (case 16) was bitten by a cat, resulting in scalp injury (table 1 ).

As shown in table 1, 11/25 cases (44\%) were from vertical transmission. Five of 11 cases (45\%) with onset before $72 \mathrm{~h}$ of life died, while no patients $(0 / 14)$ with onset after 72 h of life died (fig 1$)$. The mothers of eight cases had a positive culture for $P$ multocida in body fluid, and all had history of cats and/or dogs exposure (traumatic exposure to cats or dogs in three cases (cases 3, 10 and 13), and non-traumatic exposure in five cases (cases 6, 9, 11, 14 and 15)) (table 1). Of these, four had a positive vaginal culture (cases 10 , 11, 14 and 15), two had positive vaginal and blood cultures (cases 6 and 13), one had positive amniotic fluid culture (case 9) and one had positive endometrium culture (case 3 ). The other three cases with vertical transmission (cases 2, 22 and 25) had no evidence of maternal infection, but without evidence of animal exposure in the neonates before the onset of within $72 \mathrm{~h}$ of age. Of these, only the mother of case 25 had history of exposure to dog.

\section{CLINICAL PRESENTATIONS}

The clinical presentations of neonatal pasteurellosis were typical for neonatal sepsis. The common signs and symptoms were fever (20/ $25,80 \%)$, and irritability/lethargy (18/25, $72 \%)$. The most common clinical diagnosis was bacteraemia with meningitis $(12 / 25$ cases, $48 \%$ ), followed by bacteraemia only in four cases $(16.0 \%)$, and meningitis only in four cases $(16.0 \%)$. Other sites of infection were pneumonia (three cases), osteomyelitis (two cases) and conjunctivitis (one case) (table 2). Meningitis was found in 13 of 14 (93\%) cases with age of onset of $\geqslant 72 \mathrm{~h}$, significantly higher than in those with age of onset of $<72 \mathrm{~h}(3 / 11$ cases, $27 \%$, $p=0.002)$.

\section{LABORATORY DIAGNOSIS}

A white blood cell (WBC) count was reported in 19 cases (median: $10690 \mathrm{cell} / \mathrm{mm}^{3}$, range: $1500-35600 \mathrm{cell} / \mathrm{mm}^{3}$ ), and the median percentage of polymorphonuclear cells was 59\% (range: 10-84\%). Of the 20 cases that reported having blood culture, 17 were positive and three were negative. Five cases were not mentioned.

Of 25 reported cases, 18 underwent lumbar puncture, while four (cases 1, 6, 13 and 25) were not reported, and three (cases 3, 9 and 11) died without lumbar puncture. Of these, two had normal cerebrospinal fluid (CSF) examination (cases 14 and 22), and 16 were diagnosed with meningitis. The detailed CSF examinations were available in 13 diagnosed meningitis cases. The median WBC 


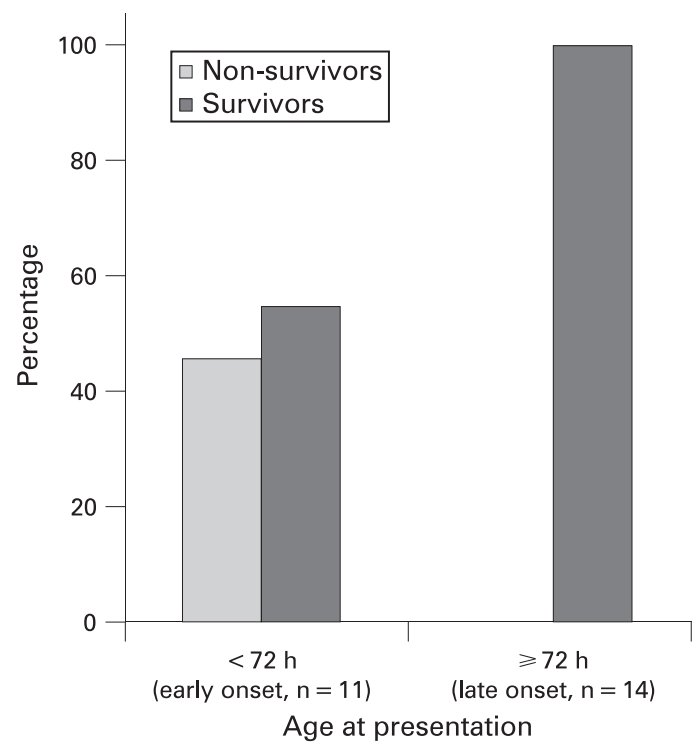

Figure 1 Distribution of age at presentation with early and late onset of neonatal pasteurellosis $(n=25)$.

count in CSF was 4780 cells $/ \mathrm{mm}^{3}$ (range: 0-11 200 cells $/ \mathrm{mm}^{3}$ ), and the median percentage of polymorphonuclear cells was $83 \%$ (range: 0-92\%). Gram stain findings of CSF specimens revealed Gram-negative coccobacilli or bacilli in 10 cases. The organism was isolated from CSF in 14/16 (88\%) cases. Another two cases (cases 19 and 21) reported a pleocytosis with negative CSF culture probably due to partial treatment. One case (case 12 ) reported no WBC in CSF but had a positive CSF culture for $P$ multocida.

\section{TREATMENT}

Treatment of $P$ multocida infection in reported cases was mainly antibiotic therapy. Surgical debridement of the brain and fractured skull was required in one case (case 8) following a penetrating injury from a dog bite. The doses and durations of antibiotic therapy of reported cases were not specified. The most frequently used antibiotics were ampicillin (16 cases), aminoglycoside (16 cases) and cefotaxime (11 cases) (table 1$)$.

\section{OUTCOME}

Of the 20 survived cases, 11 cases (case 4, 7, 8, 15, 16, 18, 19, 20, 21,24 and 25) were completely recovered at the end of followup (4-22 months), four cases (case 5, 6, 10 and 12) reported clinical normal at discharge from hospital, and five cases (case 1, 13, 14, 17 and 23) were not reported.

Five cases died, resulting in an overall mortality rate of $20 \%$ (table 2). In univariate analysis of factors potentially associated with death, age at presentation of $<72 \mathrm{~h}$, birth weight of $<2500 \mathrm{~g}$, and vertical transmission were found to be associated with death ( $p=0.009,0.009$ and 0.011 , respectively, table 3 ).

\section{DISCUSSION}

This is the first published review of pasteurellosis in neonates. We found that $P$ multocida was the only species reported. Most cases presented with bacteraemia with or without meningitis. The mortality rate of neonatal pasteurellosis was $20 \%$, and the age at presentation, birth weight, and vertical transmission were significantly associated with death.

Pasteurella spp is known to infect humans, and $P$ multocida is the most common pathogen organism. ${ }^{128}$ Diagnosis of pasteurellosis
Table 2 Characteristics of 25 reviewed cases of neonatal pasteurellosis

\begin{tabular}{|c|c|}
\hline Parameters & No of patients (\%) \\
\hline Median age at presentation (range) & $\begin{array}{l}14 \text { days (birth- } \\
30 \text { days) }\end{array}$ \\
\hline Median birth weight (range) $(\mathrm{n}=20)^{*}$ & $\begin{array}{l}3180 \mathrm{~g}(1050- \\
4300 \mathrm{~g})\end{array}$ \\
\hline \multicolumn{2}{|l|}{ Sex } \\
\hline Male & $14(56 \%)$ \\
\hline Female & $7(28 \%)$ \\
\hline Unknown & $4(16 \%)$ \\
\hline \multicolumn{2}{|l|}{ Clinical presentations $\dagger$} \\
\hline Fever & $20(80 \%)$ \\
\hline Irritability/lethargy & $18(72 \%)$ \\
\hline Apnoea, tachypnoea, dyspnoea & $11(44 \%)$ \\
\hline $\begin{array}{l}\text { Seizure, tensed anterior fontanelle, stiff } \\
\text { neck }\end{array}$ & $11(44 \%)$ \\
\hline Feeding difficulty & $10(40 \%)$ \\
\hline Anaemia, thrombocytopenia & $4(16 \%)$ \\
\hline Bradycardia, tachycardia & $3(12 \%)$ \\
\hline Hepatomegaly & $3(12 \%)$ \\
\hline Other: & $5(20 \%)$ \\
\hline \multicolumn{2}{|l|}{$\begin{array}{l}\text { Diagnostic methods (number of positive } \\
\text { culture/total cultures) }\end{array}$} \\
\hline Blood culture & $17 / 20(85 \%)$ \\
\hline Cerebrospinal fluid culture & $14 / 18(78 \%)$ \\
\hline Nasopharynx/pharynx culture & $3 / 4(75 \%)$ \\
\hline Other§ & 6 \\
\hline \multicolumn{2}{|l|}{ Clinical diagnosis $(\mathrm{n}=25)$} \\
\hline Bacteraemia and meningitis & $11(44 \%)$ \\
\hline Bacteraemia & $4(16 \%)$ \\
\hline Meningitis & $4(16 \%)$ \\
\hline Pneumonia & $2(8 \%)$ \\
\hline Bacteraemia, meningitis and osteomyelitis & $1(4 \%)$ \\
\hline Bacteraemia and pneumonia & $1(4 \%)$ \\
\hline Osteomyelitis & $1(4 \%)$ \\
\hline Conjunctivitis & $1(4 \%)$ \\
\hline \multicolumn{2}{|l|}{ Modes of transmission $(n=25)$} \\
\hline Vertical & $11(44 \%)$ \\
\hline \multicolumn{2}{|l|}{ Non-vertical } \\
\hline Non-traumatic animal exposure & $11(44 \%)$ \\
\hline Traumatic animal exposure & $2(8 \%)$ \\
\hline Unknown & $1(4 \%)$ \\
\hline \multicolumn{2}{|l|}{ Outcomes $(n=25)$} \\
\hline Died & $5(20 \%)$ \\
\hline Survived & $20(80 \%)$ \\
\hline
\end{tabular}

\footnotetext{
*Data were not available for five cases.

†Patient counts total to more than $\mathrm{n}$ values because patients with clinical presentation at $>1$ are counted more than once. flncludes eye discharge (one case), jaundice (two cases), skull fracture (one case) and skin rash (one case).

§lncludes cultures of eye discharge (case 6), placenta and gastric fluid (case 13), umbilicus (case 5), lung tissue (case 11) and pus (case 1).

Tase 8 had meningitis and brain abscess.
}

required isolation of the organism from cultures. Pasteurella grows readily on blood agar and generally appears as Gram-negative coccobacilli, therefore, was sometimes mistaken for Hemophilus spp. ${ }^{29} P$ multocida was the cause of $75-100 \%$ of cat bite wound infection and $50-55 \%$ of dog bite wound infection. ${ }^{2}{ }^{28}$ Half of all neonatal reported cases had a history of exposure to domestic animals. We found that non-traumatic exposure was more common than traumatic exposure in neonatal infection. We hypothesise that contact of respiratory droplets from animals was likely the route of infection in non-traumatic exposure cases. The organism has been known to be transmitted through animal saliva without a bite or scratch. ${ }^{30}$ 
Table 3 Univariate analysis for risk factors associated with death in neonatal pasteurellosis

\begin{tabular}{llll}
\hline & \multicolumn{3}{l}{ Outcome, $\mathbf{n}(\%)$} \\
\cline { 2 - 4 } Variables & $\begin{array}{l}\text { Survivors } \\
(\mathbf{n}=\mathbf{2 0})\end{array}$ & $\begin{array}{l}\text { Non- } \\
\text { survivors } \\
(\mathbf{n}=\mathbf{5})\end{array}$ & $\mathbf{p}$ Value \\
\hline Age at presentation $<72 \mathrm{~h}$ & $6 / 20(30)$ & $5 / 5(100)$ & 0.009 \\
Birth weight $<2500 \mathrm{~g}^{*}$ & $0 / 15(0)$ & $3 / 5(60)$ & 0.009 \\
Male $\dagger$ & $9 / 16(56)$ & $5 / 5(100)$ & 0.123 \\
Vertical transmission $\uparrow$ & $6 / 19(32)$ & $5 / 5(100)$ & 0.011 \\
\hline
\end{tabular}

Data were not available for $5^{*}, 4 \dagger$ and $1+$ cases.

Vertical transmission defined as documented evidence of Pasteurella spp infection in organ(s) and/or body fluid in the mothers, and/or the patients developed pasteurellosis within $72 \mathrm{~h}$ of age and without history of neonatal exposure to animals.

This review showed that vertical transmission is an important route of infection in the neonatal population, and was a significant factor associated with death. This study found that eight of 10 mothers of infants with vertical transmission had $P$ multocida infections in blood and/or genital tract fluid including placenta. Transplacental haematogenous infection, particularly intrauterine transmission, and infection in the mother at time at birth can play a significant role in the development of infection in the neonates. ${ }^{31}{ }^{32}$

The presenting features of $P$ multocida infection in neonates were sepsis-like. Similar to other neonatal bacterial sepsis, neonatal pasteurellosis could be classified into early- and lateonset forms. The results of this study revealed that early onset (age at presentation within $72 \mathrm{~h}$ of age) was associated with death, and less likely involved the central nervous system (CNS) than late onset. The neonates with early onset had more severe infection and might have died before the lumbar puncture was performed, and missed the diagnosis of meningitis. The neonates with late onset, could be from a smaller inoculum amount of bacteria, had a longer time to offset the infection and allow dissemination of bacteria to multiple organs including the CNS, similar to other late-onset neonatal sepsis. ${ }^{31}$ When compared with a report of Kimura et $a l,{ }^{33}$ in which the majority of patients were adults, the proportion of CNS infections in neonates was higher ( $64 \%$ vs $14 \%$, respectively). Cellulitis was the most common clinical presentation in adults. Immature immunity could explain the more severe form of infections in neonates. ${ }^{3132} 34$

The antibiotic therapy of choice for pasteurellosis in children is penicillin. In neonates, a high dose of penicillin should be used to treat CNS infections. Aminoglycoside should also be administered for synergistic mechanisms. The recommended duration of treatment was 7-10 days for local infections, and extended to 2 weeks for neonatal bacteraemia and meningitis. Treatment of bone and/or joint infections should be 4-6 weeks. Surgical drainage or debridement may be necessary in patients with site abscess or bite wound. ${ }^{35}$

Our study found that age at presentation of $<72 \mathrm{~h}$, birth weight of $<2500 \mathrm{~g}$, and vertical transmission were independent factors associated with mortality. This result was similar to other bacterial neonatal sepsis in that early-onset and low birth weight infants had a higher mortality rate. ${ }^{31}{ }^{32}$ Furthermore, vertical infection may have a higher inoculum size and therefore cause a more serious infection.

In conclusion, neonatal pasteurellosis was a rare infectious disease, but with a relatively high mortality rate. We hope this report alerts general paediatricians and neonatologists to consider pasteurellosis as a possible cause of sepsis in neonates, especially in patients and mothers with a history of domestic animal exposure.
Acknowledgements: The authors thank Mr David Patterson for editing the manuscript.

Competing interests: None.

\section{REFERENCES}

1. Weber DJ, Wolfson JS, Swart MN, et al. Pasteurella multocida infections: report of 34 cases and review of the literature. Medicine (Baltimore) 1984;63:133-54.

2. Donnio PY, Lerestif-Gautier AL, Avril JL. Characterization of Pasteurella spp. strains isolated from human infections. J Comp Pathol 2004;130:137-42.

3. Pizey NCD. Infection with Pasteurella septica in a child aged three weeks. Lancet 1953;265:324-6.

4. Bates HA, Controni G, Elliott N, et al. Septicemia and meningitis in a newborn due to Pasteurella multocida. Clin Pediatr 1965;4:668-70.

5. Strand CL, Helfman L. Pasteurella multocida chorioamnionitis associated with premature delivery and neonatal sepsis and death. Am J Clin Pathol 1971;55:713-6.

6. Repice JP, Neter E. Pasteurella multocida meningitis in an infant with recovery. $J$ Pediatr 1975;86:91-3.

7. Frutos AA, Levitsky D, Scott EG, et al. A case of septicemia and meningitis in an infant due to Pasteurella multocida. J Pediatr 1978;92:853.

8. Khan MS, Stead SE. Neonatal Pasteurella multocida conjunctivitis following zoonotic infection of mother. J Infect 1979;1:289-90.

9. Thompson CM, Pappu L, Levkoff AH, et al. Neonatal septicemia and meningitis due to Pasteurella multocida. Pediatr Infect Dis J 1984;3:559-61.

10. Steinbok P, Flodmark O, Scheifele DW. Animal bites causing central nervous system injury in children. Pediatr Neurosci 1985-1986;12:69-100.

11. Wong GP, Cimolai N, Dimmick JE, et al. Pasteurella multocida chorioamnionitis from vaginal transmission. Acta Obstet Gynecol Scand 1992;11:384-7.

12. Hillery S, Reiss-Levy EA, Browne C, et al. Pasteurella multocida meningitis in a twoday old neonate. Scand J Infect Dis 1993;25:655-8.

13. Andersson S, Larinkari U, Vartia T, et al. Fatal congenital pneumonia caused by catderived Pasteurella multocida. Pediatr Infect Dis J 1994;13:74-5.

14. Miller JJ, Gray BM. Pasteurella multocida meningitis presenting as fever with a source in a young infant. Pediatr Infect Dis J 1995;14:331-2.

15. Escande $\mathbf{F}$, Borde $\mathrm{M}$, Pateyron $\mathrm{F}$. Infection maternelle et néonatale à Pasteurella multocida. Arch Pédiatr 1997:4:1116-8.

16. Challapilla M, Convert RF. Infectious diseases case book. Pasteurella multocida early onset septicemia in newborns. J Perinatol 1997;17:248-9.

17. Zaramella $\mathbf{P}$, Zamorani E, Freato F. Neonatal meningitis due to a vertical transmission of Pasteurella multocida. Pediatr Int 1999:41:307-10.

18. Kouppari G, Garoufi A, Stamos G, et al. Pasteurella multocida septicemia and meningitis in an infant. Clin Microbiol Infect 1999;5:101-2.

19. Boerlin $\mathbf{P}$, Siegrist HH, Burnens AP, et al. Molecular identification and epidemiological tracing of Pasteurella multocida meningitis in a baby. J Clin Microbiol 2000;38:1235-7.

20. Mestre F, Laluque $\mathrm{S}$, Gallet $\mathrm{S}$, et al. Méningitis néonatale à Pasteurella multocida. Arch Pédiatr 2001;22:670-1.

21. Hirsh D, Farrell K, Reilly C, et al. Pasteurella multocida meningitis and cervical spine osteomyelitis in a neonate. Pediatr Infect Dis J 2004;23:1063-5.

22. Best EJ, Lahra MM, Palasanthiran P. An unusual neonatal zoonosis. Med J Aus 2005; 182:137.

23. Cohen-Adam D, Marcus N, Scheuerman O, et al. Pasteurella multocida septicemia in a newborn without scratches, licks or bites. Isr Med Assoc J 2006:8:657-8.

24. Puwanant M, Chanvitan P. Neonatal septicemia due to Pasteurella multocida: the first case report in Thailand. J Med Assoc Thai 2006;89:1293-6.

25. Goytia VK, Demmler GJ, Pannaraj PS, et al. An unusual cause of sepsis and meningitis in a neonate. Semin Pediatr Infect Dis 2006;17:187, 225-7.

26. Haase R, Stiefel M, Merkel N, et al. Pasteurella multocida as a rare cause of neonatal meningitis: case report and review of the literature. Z Geburtsh Neonatol 2006;210:219-21

27. Pace D, Attard-Montalto S. Case 1: a neonatal zoonosis. Acta Paediatr 2008;97:250-4

28. Talan DA, Citron DM, Abrahamian FM, et al. Bacteriologic analysis of infected dog and cat bites. N Engl J Med 1999;340:85-92.

29. Hamilton-Miller JMT. A possible pitfall in the identification of Pasteurella spp. with the API system. J Med Microbiol 1993;39:78-9.

30. Costa-Cruz 0, Sesso AM, Mate S, et al. Pasteurella multocida meningitis. N Jersey Med 1990;87:127-9.

31. Edward MS, Baker CJ. Sepsis in the newborn. In: Gershon AA, Hotez PJ, Katz SL, eds. Infectious diseases of children. 8th edn. Philadelphia: Mosby, 2004: 545-62.

32. Palazzi DL, Klein JO, Baker CJ. Bacterial sepsis and meningitis. In: Remington JS, Klein JO, Wilson CB, eds. Infectious diseases of the fetus and newborn infants. 6th edn. Philadelphia: Elsevier-Sounders, 2006: 247-96.

33. Kimura R, Hayashi $Y$, Takeuchi T, et al. Pasteurella multocida septicemia caused by close contact with a domestic cat: case report and literature review. J Infect Chemother 2004;10:250-2.

34. Bellanti JA, Zeligs BJ, Pung YH. Immunology of the fetus and newborn. In: MacDonald MG, Seshia MMK, Mullett MD, eds. Avery's neonatology: pathophysiology \& management of the newborn. 6th edn. Philadelphia: Lippincott William \& Wilkins, 2005: 1135-61.

35. American Academy of Pediatrics. Pasteurella infection. In: Pickering LK, Baker CJ, Long SS, eds. Redbook: 2006 report of the committee on infectious diseases. 27th edn. Elk Grove Village, Illinois: American Academy of Pediatrics, 2006: 487-8. 